\title{
ARTICLE
}

Molecular Diagnostics

\section{Prognostic value of serum alkaline phosphatase in spinal metastatic disease}

\author{
Aditya V. Karhade ${ }^{1}$, Quirina C. B. S. Thio ${ }^{1}$, Megna Kuverji ${ }^{1}$, Paul T. Ogink ${ }^{1}$, Marco L. Ferrone ${ }^{2}$ and Joseph H. Schwab ${ }^{1}$
}

BACKGROUND: Determination of the appropriateness of invasive management in patients with spinal metastatic disease requires accurate pre-operative estimation of survival. The purpose of this study was to examine serum alkaline phosphatase as a prognostic marker in spinal metastatic disease.

METHODS: Chart reviews from two tertiary care centres were used to identify spinal metastatic disease patients. Bivariate and multivariate analyses were used to determine if serum alkaline phosphatase was an independent prognostic marker for survival. RESULTS: Overall, 732 patients were included with 90-day and 1-year survival of $n=539(74.9 \%)$ and $n=324$ (45.7\%), respectively. The 1-year survival of patients in the first quartile of alkaline phosphatase ( $\leq 73 \mathrm{IU} / \mathrm{L}$ ) was $78(57.8 \%)$ compared to 31 (24.0\%) for patients in the fourth quartile ( $>140 \mathrm{lU} / \mathrm{L})$. Preoperative serum alkaline phosphatase levels were significantly elevated in patients with multiple spine metastases, non-spine bone metastasis, and visceral metastasis but not in patients with brain metastasis. On multivariate analysis, elevated serum alkaline phosphatase was identified as an independent prognostic factor for survival in spinal metastatic disease.

CONCLUSION: Serum alkaline phosphatase is associated with preoperative metastatic tumour burden and is a biomarker for overall survival in spinal metastatic disease.

British Journal of Cancer (2019) 120:640-646; https://doi.org/10.1038/s41416-019-0407-8

\section{BACKGROUND}

Spinal metastases are the most common type of bone metastasis and have a prevalence of $30-50 \%$ in cancer patients. ${ }^{1-3}$ Spinal metastases lead to spinal instability, pathologic fractures, neurologic deficits, and decreased quality of life. ${ }^{1,2}$ Management of spinal metastases is primarily palliative and includes consideration of surgery, radiotherapy, medical management, and palliative therapy. ${ }^{4}$ Determination of the appropriateness of invasive management such as multi-level decompression and stabilisation requires accurate pre-operative estimation of survival. ${ }^{4,5}$ A number of prognostic factors have been identified in this population but routinely collected laboratory markers have yet to be fully understood or utilised. ${ }^{5-7}$ Serum alkaline phosphatase is one such marker that is routinely collected in spinal metastatic disease patients but remains underutilised for prognostication.

Alkaline phosphatase has been well-established as a marker of hepatobiliary pathology and bone turnover and mineralisation. $^{8-11}$ This metalloenzyme is expressed on the cell surface of osteoblasts and serum levels of the enzyme correlate with increased osteoblastic activity. ${ }^{10,12}$ In osteolytic bone metastases this enzyme is elevated secondary to a local bone formation response in an attempt to compensate for the predominant destructive lesion. ${ }^{10}$ In osteoblastic bone metastases, alkaline phosphatase is elevated secondary to local stimulation of osteoblasts. ${ }^{10}$ In visceral metastasis, serum alkaline phosphatase is elevated secondary to intrahepatic biliary tract obstruction by hepatic metastatic tumour burden. ${ }^{11}$ On the basis of this known pathophysiology, we hypothesised that elevated serum alkaline phosphatase would be a marker for survival in spinal metastatic disease as an aggregate measure for metastatic tumour burden.

As such, the primary purpose of this study was to determine if serum alkaline phosphatase was an independent prognostic factor for survival in spinal metastatic disease. The secondary aim of this study was to characterise the relationship between serum alkaline phosphatase and metastatic tumour burden.

\section{MATERIALS AND METHODS}

Guidelines

This study followed the Reporting Recommendations for Tumour Marker Prognostic studies (REMARK) guidelines and the Transparent Reporting of a Multivariable Prediction Model for Individual Prognosis or Diagnosis (TRIPOD) guidelines. ${ }^{13,14}$

\section{Data source}

Chart review was conducted at two tertiary care centres to identify patients with a diagnosis of secondary malignant neoplasm of bone or pathological fracture in metastatic disease. Clinical records for these patients were retrospectively reviewed to ascertain the following inclusion criteria: (1) age >18 years, (2) diagnosis of spinal metastatic disease, (3) initial decompression or

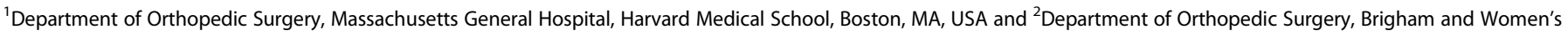
Hospital, Harvard Medical School, Boston, MA, USA

Correspondence: Joseph H. Schwab (jhschwab@mgh.harvard.edu)

Received: 11 September 2018 Revised: 31 January 2019 Accepted: 1 February 2019

Published online: 22 February 2019 
stabilisation between 1 January 2000 and 31 December 2016. This study was approved by our institutional review board.

\section{Outcomes}

Post-operative all-cause mortality was the primary outcome in this study. The Social Security Death index was available up to 2014 and was used to establish mortality for this time-period and chart review was used to ascertain mortality thereafter. Ninety-day mortality could be ascertained in 720 (98.4\%) of patients and 1 -year mortality could be ascertained in 709 (96.9\%) of patients.

\section{Variables}

The following pre-operative variables were collected by chart review: age, sex, body mass index [kilograms per metre squared $\left.\left(\mathrm{kg} / \mathrm{m}^{2}\right)\right]$, preoperative presence of any Charlson comorbidity other than metastatic disease, ${ }^{15}$ primary tumour histology [based on slow, moderate, and rapid growth groupings as classified by Katagiri et al.] ${ }^{16}$ (see Appendix Supplementary Table 1 for histology included in each group). Additional factors were pathological fracture at presentation, pain at presentation, Eastern Cooperative Oncology Group performance status, American Spinal Injury Association Impairment Scale, spine tumour location, number of spinal metastases, other non-spine bone metastases, presence of visceral metastases (metastases in liver or lung), presence of brain metastases, history of local radiation to affected site, history of previous systemic therapy, preoperative serum laboratory characteristics in 30-days before surgery: white blood cell $\left[10^{3} /\right.$ microlitre $\left.(\mu \mathrm{L})\right]$, haemoglobin [grams per decilitre $\left.(\mathrm{g} / \mathrm{dL})\right]$, platelet $\left(10^{3} / \mu \mathrm{L}\right)$, absolute lymphocyte $\left(10^{3} / \mu \mathrm{L}\right)$, absolute neutrophil $\left(10^{3} / \mu \mathrm{L}\right)$, platelet to lymphocyte ratio, neutrophil to lymphocyte ratio, albumin $(\mathrm{g} / \mathrm{dL})$, alkaline phosphatase [international units per litre (IU/L), calcium [milligrams per decilitre $(\mathrm{mg} / \mathrm{dL})$ ], and creatinine $(\mathrm{mg} / \mathrm{dL})$. Operative factors assessed were: number of vertebral levels, surgical approach, surgical type (decompression, stabilisation, corpectomy).

\section{Missing data}

Multiple imputation with Stekhoven et al.'s nonparametric missForest methodology, based on the machine learning methodology of random forests, was used to impute variables with less than $30 \%$ missing data. ${ }^{17}$

\section{Statistical analysis}

Descriptive statistics were generated for the baseline characteristics of the population. Bivariate analysis with the non-parametric Mann-Whitney-Wilcoxon test was used to assess the relationship between serum alkaline phosphatase and preoperative metastatic tumour burden (multiple spine metastases, other non-spine bone metastases, visceral metastases, and brain metastases). LópezRatón et al.'s optimal cutpoint method with the area under the receiver operating curve metric was used to determine the threshold for elevated serum alkaline phosphatase. ${ }^{18}$ Kaplan Meier curves were generated for survival by strata of serum alkaline phosphatase. Bivariate Cox proportional hazards analysis was used to assess the association of baseline characteristics with overall survival. Multivariable Cox proportional hazards analysis with bootstrapped (100 replications with replacement) backward stepwise elimination was used to determine if alkaline phosphatase was an independent prognostic factor for overall survival. Sensitivity analyses were conducted by repeating the multivariable Cox proportional hazards analysis with inclusion of alkaline phosphatase as a continuous variable and alkaline phosphatase at other thresholds previously studied in the literature $(113,135 \mathrm{IU} / \mathrm{L})$. Finally, multivariable logistic regression with bootstrapped (100 replications with replacement) backward stepwise elimination was used to determine whether serum alkaline phosphatase was an independent risk factor for mortality at both 90-days and 1-year after surgery. ${ }^{19,20}$

\section{RESULTS}

Of seven hundred and thirty-two spinal metastatic disease patients included in this study, the median age was 61 (interquartile range 53-69) and 206 (41.8\%) were female (Table 1). The 30-day, 90-day, and 1-year survival for these patients were $n=662$ (91.4\%), $n=539$ (74.9\%), and $n=324$ (45.7\%), respectively.

The optimal cut-off for serum alkaline phosphatase was determined to be 100 international units per litre (IU/L) using the area under the receiver operating curve metric. Patients with alkaline phosphatase $\geq 100 \mathrm{IU} / \mathrm{L}$ had shorter predicted postoperative survival at all time points up to 1-year (Fig. 1). The 1-year survival by quartile of serum alkaline phosphatase was: 78 (57.8\%) for alkaline phosphatase $\leq 73 \mathrm{IU} / \mathrm{L}, 71$ (55.0\%) for $73 \mathrm{IU} / \mathrm{L}<$ alkaline phosphatase $\leq 94.5 \mathrm{IU} / \mathrm{L}, \quad 50 \quad(37.9 \%)$ for $94.5 \mathrm{IU} / \mathrm{L}<$ alkaline phosphatase $\leq 140 \mathrm{IU} / \mathrm{L}$, and 31 (24.0\%) for alkaline phosphatase >140 IU/L.

Metastatic tumour burden

In patients with multiple spine metastasis, $n=521$ (71.2\%), serum alkaline phosphatase was, median (interquartile range), 102 (76-154) IU/L whereas in patients with a single spine metastasis, serum alkaline phosphatase was $84(69-106) \mathrm{IU} / \mathrm{L}(p<0.001)$. In patients with other non-spine bone metastasis, $n=388$ (53\%), serum alkaline phosphatase was 105 (79-168) IU/L whereas in patients with no non-spine bone metastasis, serum alkaline phosphatase levels were $87(69-112)$ IU/L $(p<0.001)$. In patients with visceral metastasis, $n=252$ (34.4\%), serum alkaline phosphatase was 105 (80-172) IU/L whereas in patients with no visceral metastasis, serum alkaline phosphatase levels were 91 $(69-128)$ IU/L $(p<0.001)$. In patients with brain metastasis, $n=$ $81(11.1 \%)$, serum alkaline phosphatase was 100 (73-134) IU/L whereas in patients with no brain metastasis, serum alkaline phosphatase levels were 94 (73-140) IU/L $(p=0.85)$. Overall, serum alkaline phosphatase levels were significantly elevated in patients with multiple spine metastases $(p<0.001)$, non-spine bone metastasis $(p<0.001)$, and visceral metastasis $(p<0.001)$ but not in patients with brain metastasis $(p=0.85)$ (Fig. 2$)$.

\section{Survival analysis}

On bivariate Cox proportional hazards analysis, primary tumour histology, BMI, concurrent medical comorbidities, performance status, neurologic deficit, spinal location, metastatic tumour burden (multiple spine metastases, other non-spine bone metastases, visceral metastases, brain metastases), history of local radiation, history of previous systemic therapy, anaemia, thrombocytopenia, thrombocytosis, lymphocytopenia, neutrophilia, NLR, PLR, hypoalbuminemia, alkaline phosphatase, calcium, creatinine, and more invasive surgery were associated with survival (Table 2).

On multivariate Cox proportional hazards analysis, serum alkaline phosphatase $>100 \mathrm{IU} / \mathrm{L}$ remained an independent prognostic factor for overall survival (Table 3 ). On sensitivity analyses with assessment of serum alkaline phosphatase as a continuous variable and alkaline phosphatase at thresholds of $113 \mathrm{IU} / \mathrm{L}$ and $135 \mathrm{IU} / \mathrm{L}$, serum alkaline phosphatase remained an independent prognostic factor for survival [Supplementary Tables 2-4].

On multivariable logistic regression of 90-day mortality, serum alkaline phosphatase (continuous) was an independent prognostic factor [Supplementary Table 5]. On multivariable logistic regression of 1-year mortality, serum alkaline phosphatase (continuous) was an independent prognostic factor [Supplementary Table 6].

\section{DISCUSSION}

In this population of 732 patients undergoing intervention for spinal metastatic disease, serum alkaline phosphatase was significantly elevated in patients with multiple spinal metastases, other non-spine bone metastases, and visceral metastases but not in patients with brain metastases. Elevated serum alkaline 
Table 1. Baseline characteristics of study population, $n=732$

\begin{tabular}{l} 
Variable \\
\hline Age (years) \\
$<65$ \\
$>=65$ \\
Sex \\
Female \\
Male \\
BMI $\left(\mathrm{kg} / \mathrm{m}^{2}\right)$ \\
$18-30$ \\
$<18$ \\
$>30$
\end{tabular}

Not recorded

Other Charlson comorbidity

Primary Tumour Histology

Group 1 (slow growth)

Group 2 (moderate growth)

Group 3 (rapid growth)

Pathological fracture

Pain

ECOG

$0-2$

3-4

Not recorded

ASIA

Normal (E)

Impaired (A-D)

Not recorded

Tumour location

Cervical

Thoracic

Lumbar

Multiple

Spine metastases

One

Two

Three or more

Other bone metastases

Visceral metastases

Brain metastases

History of local radiation

Previous systemic therapy

White blood cell $\left(10^{3} / \mu \mathrm{L}\right)$

$<11$

$>=11$

Not measured

Haemoglobin $(\mathrm{g} / \mathrm{dL})$

$<13$

$>=13$

Not measured

Platelet $\left(10^{3} / \mu \mathrm{L}\right)$

$<150$

$>450$

150-450

Not measured $n(\%)$

448 (61.2)

284 (38.8)

$306(41.8)$

426 (58.2)

471 (64.3)

21 (2.9)

150 (20.5)

$90(12.3)$

441 (60.2)

219 (29.9)

254 (34.7)

259 (35.4)

456 (62.3)

627 (85.7)

$440(60.1)$

99 (13.5)

193 (26.4)

379 (51.8)

342 (46.7)

11 (1.5)

104 (14.2)

425 (58.1)

164 (22.4)

$39(5.3)$

211 (28.8)

117 (16.0)

404 (55.2)

388 (53.0)

252 (34.4)

81 (11.1)

252 (34.4)

418 (57.1)

486 (66.4)

157 (21.4)

89 (12.2)

435 (59.4)

208 (28.4)

89 (12.2)

75 (10.2)

50 (6.8)

$518(70.8)$

89 (12.2)
Table 1 continued

\begin{tabular}{|c|c|}
\hline Variable & $n(\%)$ \\
\hline \multicolumn{2}{|c|}{ Absolute lymphocyte $\left(10^{3} / \mu \mathrm{L}\right)$} \\
\hline$<1$ & $280(38.3)$ \\
\hline$>=1$ & $234(32.0)$ \\
\hline Not measured & $218(29.8)$ \\
\hline \multicolumn{2}{|c|}{ Absolute neutrophil $\left(10^{3} / \mu \mathrm{L}\right)$} \\
\hline$<6$ & $232(31.7)$ \\
\hline$>=6$ & $285(38.9)$ \\
\hline Not measured & $215(29.4)$ \\
\hline \multicolumn{2}{|c|}{ Neutrophil to lymphocyte ratio } \\
\hline$<4.7$ & $174(23.8)$ \\
\hline$>=4.7$ & $340(46.4)$ \\
\hline Not measured & $218(29.8)$ \\
\hline \multicolumn{2}{|c|}{ Platelet to lymphocyte ratio } \\
\hline$<408$ & $355(48.5)$ \\
\hline$>=408$ & $159(21.7)$ \\
\hline Not measured & $218(29.8)$ \\
\hline \multicolumn{2}{|l|}{ Albumin $(\mathrm{g} / \mathrm{dL})$} \\
\hline$<3.5$ & $156(21.3)$ \\
\hline$>=3.5$ & $391(53.4)$ \\
\hline Not measured & $185(25.3)$ \\
\hline \multicolumn{2}{|c|}{ Alkaline phosphatase (IU/L) } \\
\hline$<100$ & 290 (39.6) \\
\hline$>=100$ & $248(33.9)$ \\
\hline Not measured & $194(26.5)$ \\
\hline \multicolumn{2}{|l|}{ Calcium (mg/dL) } \\
\hline$<9$ & $257(35.1)$ \\
\hline$>=9$ & $365(49.9)$ \\
\hline Not measured & $110(15.0)$ \\
\hline \multicolumn{2}{|l|}{ Creatinine (mg/dL) } \\
\hline$<1$ & $467(63.8)$ \\
\hline$>=1$ & $176(24.0)$ \\
\hline Not measured & $89(12.2)$ \\
\hline \multicolumn{2}{|c|}{ Number of levels operated } \\
\hline One or two & $531(72.5)$ \\
\hline Three or more & $200(27.3)$ \\
\hline Anterior approach & $105(14.3)$ \\
\hline Posterior approach & $660(90.2)$ \\
\hline Combined approach & $33(4.5)$ \\
\hline Decompression & $699(95.5)$ \\
\hline Stabilisation & $640(87.4)$ \\
\hline Corpectomy & $351(48.0)$ \\
\hline
\end{tabular}

phosphatase was identified as an independent prognostic factor for survival on multivariate Cox proportional hazard analyses and further confirmed as a prognostic factor at both 90-days and 1-year after surgery on multivariate logistic regression analyses.

In metastatic disease, alkaline phosphatase has been previously examined as a diagnostic marker for the presence of liver and bone metastases. Tartter et al. ${ }^{21}$ retrospectively studied 327 patients with colorectal cancer and found sensitivity of $88 \%$ and false positive rate of $12 \%$ for the presence of liver metastases with 


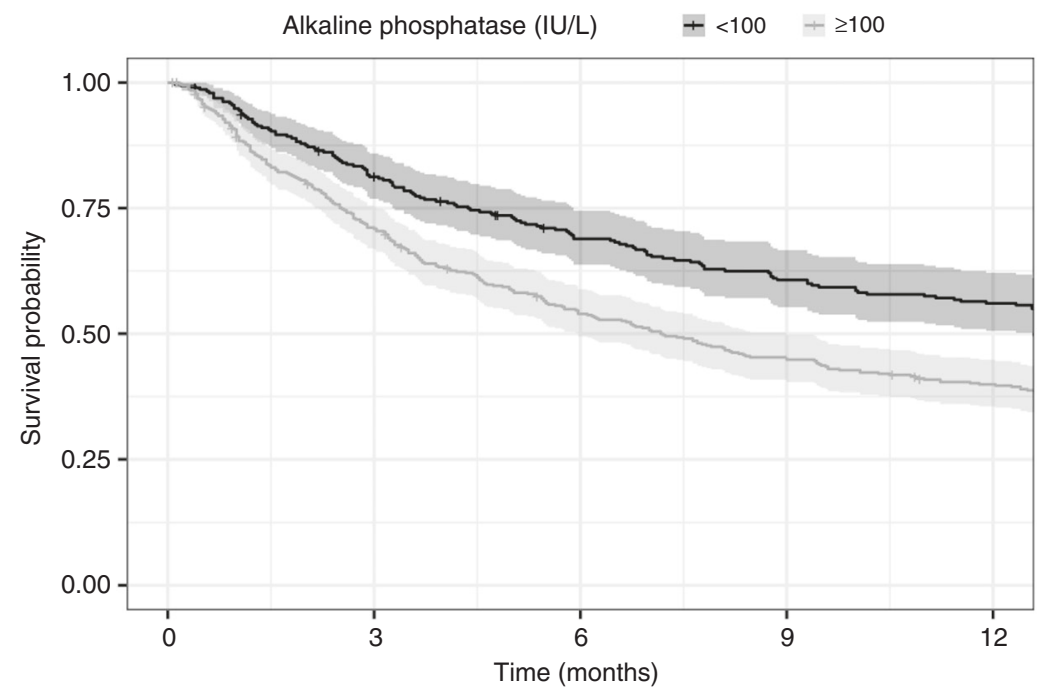

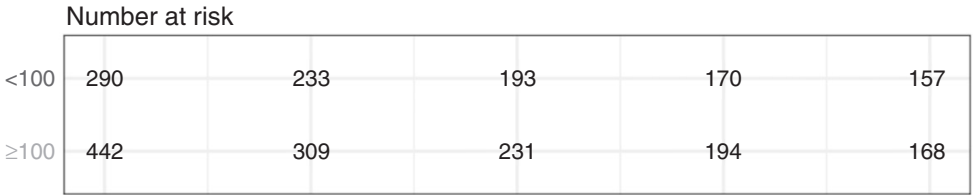

Fig. 1 Kaplan-Meier curve by strata of serum alkaline phosphatase. Patients with elevated preoperative serum alkaline phosphatase had lower predicted survival at all time points up to 1-year
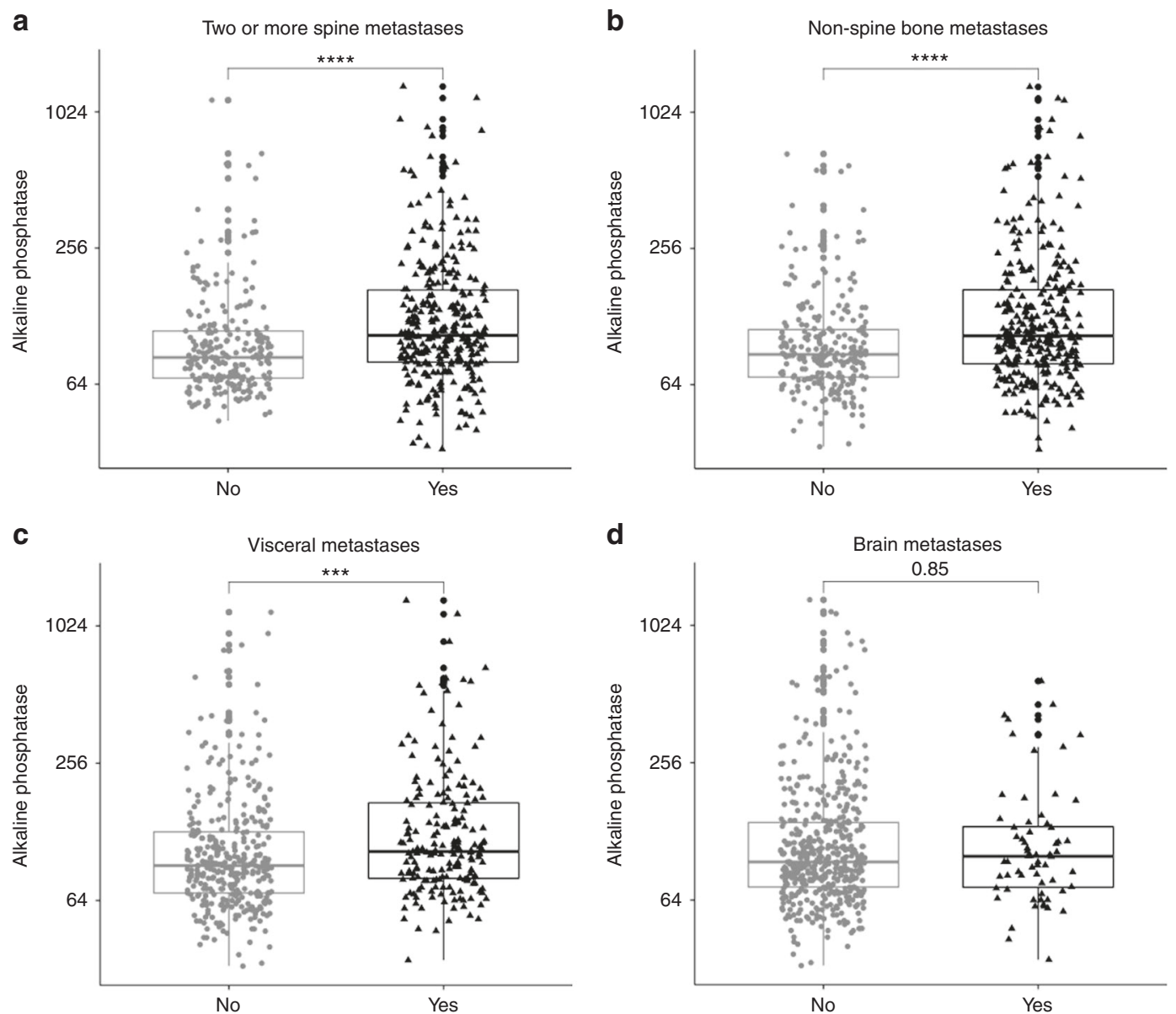

Fig. 2 a-c Serum alkaline phosphatase was associated with preoperative burden of multiple spine metastases, non-spine metastases, and visceral metastases $\left({ }^{* *} p<0.001\right)$. d Serum alkaline phosphatase was not associated with preoperative burden of brain metastases $(p=0.85)$ 
Table 2. Bivariate Cox proportional hazards regression analyses of baseline characteristics, $n=732$

\begin{tabular}{|c|c|c|c|}
\hline Variable & Odds ratio & $95 \% \mathrm{Cl}$ & $p$-value \\
\hline Age (years) & 1.07 & $(0.90,1.26)$ & 0.45 \\
\hline Female sex & 1.00 & $(0.85,1.18)$ & 0.98 \\
\hline \multicolumn{4}{|l|}{ BMI $\left(\mathrm{kg} / \mathrm{m}^{2}\right)$} \\
\hline $18-30$ & Reference & - & - \\
\hline$<18$ & 1.83 & $(1.14,2.95)$ & 0.01 \\
\hline$>30$ & 0.85 & $(0.69,1.04)$ & 0.12 \\
\hline Other Charlson comorbidity & 1.26 & $(1.06,1.49)$ & 0.007 \\
\hline \multicolumn{4}{|l|}{ Primary Tumour Histology } \\
\hline Group 1 (slow growth) & Reference & - & - \\
\hline Group 2 (moderate growth) & 1.74 & $(1.40,2.15)$ & $<0.001$ \\
\hline Group 3 (rapid growth) & 3.21 & $(2.60,3.98)$ & $<0.001$ \\
\hline Pathological fracture & 1.13 & $(0.96,1.34)$ & 0.14 \\
\hline Pain & 0.97 & $(0.78,1.22)$ & 0.8 \\
\hline ECOG 3-4 & 2.65 & $(2.10,3.35)$ & $<0.001$ \\
\hline ASIA Impaired (A-D) & 1.5 & $(1.28,1.77)$ & $<0.001$ \\
\hline Cervical Metastasis & 1.09 & $(0.86,1.38)$ & 0.47 \\
\hline Thoracic Metastasis & 1.21 & $(1.02,1.43)$ & 0.03 \\
\hline Lumbar Metastasis & 0.74 & $(0.61,0.91)$ & 0.004 \\
\hline Two or More Spine Metastases & 1.64 & $(1.36,1.98)$ & $<0.001$ \\
\hline Other Bone Metastases & 1.43 & $(1.21,1.68)$ & $<0.001$ \\
\hline Visceral Metastases & 1.77 & $(1.49,2.10)$ & $<0.001$ \\
\hline Brain Metastases & 2.07 & $(1.63,2.63)$ & $<0.001$ \\
\hline History of local radiation & 1.23 & $(1.04,1.46)$ & 0.02 \\
\hline Previous systemic therapy & 1.78 & $(1.50,2.11)$ & $<0.001$ \\
\hline White Blood Cell $\left(10^{3} / \mu \mathrm{L}\right)>=11$ & 1.15 & $(0.94,1.40)$ & 0.17 \\
\hline Haemoglobin $(\mathrm{g} / \mathrm{dL})<13$ & 1.68 & $(1.39,2.04)$ & $<0.001$ \\
\hline \multicolumn{4}{|l|}{ Platelet $\left(\times 10^{3} / \mu \mathrm{L}\right)$} \\
\hline $150-450$ & Reference & - & - \\
\hline$<150$ & 1.47 & $(1.13,1.92)$ & 0.004 \\
\hline$>450$ & 1.44 & $(1.05,1.97)$ & 0.02 \\
\hline Absolute lymphocyte $\left(10^{3} / \mu \mathrm{L}\right)<1$ & 1.60 & $(1.32,1.94)$ & $<0.001$ \\
\hline Absolute neutrophil $\left(10^{3} / \mu \mathrm{L}\right)>6$ & 1.28 & $(1.05,1.55)$ & 0.01 \\
\hline $\begin{array}{l}\text { Neutrophil to lymphocyte ratio } \\
>=4.7\end{array}$ & 1.80 & $(1.46,2.22)$ & $<0.001$ \\
\hline Platelet to lymphocyte ratio $>=408$ & 1.66 & $(1.36,2.04)$ & $<0.001$ \\
\hline Albumin $(\mathrm{g} / \mathrm{dL})<3.5$ & 1.97 & $(1.61,2.41)$ & $<0.001$ \\
\hline $\begin{array}{l}\text { Alkaline phosphatase (IU/L) >= } \\
100\end{array}$ & 1.84 & $(1.52,2.22)$ & $<0.001$ \\
\hline Calcium $(\mathrm{mg} / \mathrm{dL})>=9$ & 0.73 & $(0.62,0.88)$ & $<0.001$ \\
\hline Creatinine $(\mathrm{mg} / \mathrm{dL})>=1$ & 0.77 & $(0.64,0.94)$ & 0.009 \\
\hline Number of levels operated & 1.16 & $(0.96,1.40)$ & 0.12 \\
\hline Anterior approach & 0.75 & $(0.60,0.95)$ & 0.02 \\
\hline Posterior approach & 1.12 & $(0.86,1.47)$ & 0.41 \\
\hline Combined approach & 0.57 & $(0.37,0.87)$ & 0.01 \\
\hline Decompression & 1.23 & $(0.81,1.86)$ & 0.34 \\
\hline Stabilisation & 0.74 & $(0.59,0.94)$ & 0.01 \\
\hline Corpectomy & 0.86 & $(0.73,1.02)$ & 0.08 \\
\hline
\end{tabular}

ASIA American Spinal Injury Association Impairment Scale, $B M I$ body mass index, ECOG Eastern Cooperative Oncology Group performance status, $(g / d L)$ grams per decilitre, $(I U / L)$ international units per litre, $(m g / d L)$ milligrams per decilitre, $\mu L$ microlitre

Serum alkaline phosphatase provided in bold
Table 3. Multivariate Cox-proportional hazards regression, $n=732$

\begin{tabular}{lllc}
\hline Variable & HR & $95 \% \mathrm{Cl}$ & $p$-value \\
\hline Other Charlson comorbidity & 1.21 & $(1.02,1.43)$ & 0.03 \\
Primary Tumour Histology & & & \\
$\quad$ Group 1 (slow growth) & Reference & - & - \\
$\quad$ Group 2 (moderate growth) & 1.55 & $(1.25,1.94)$ & $<0.001$ \\
$\quad$ Group 3 (rapid growth) & 2.99 & $(2.38,3.75)$ & $<0.001$ \\
ECOG 3-4 & 2.66 & $(2.14,3.31)$ & $<0.001$ \\
Two or more spine metastases & 1.36 & $(1.11,1.65)$ & 0.002 \\
Other bone metastases & 1.24 & $(1.04,1.49)$ & 0.02 \\
Visceral metastases & 1.17 & $(0.97,1.41)$ & 0.09 \\
Brain metastases & 1.43 & $(1.12,1.83)$ & 0.004 \\
Previous systemic therapy & 1.42 & $(1.19,1.7)$ & $<0.001$ \\
Hemoglobin (g/dL) $<13$ & 1.38 & $(1.13,1.67)$ & 0.001 \\
Platelet to lymphocyte ratio $>=408$ & 1.17 & $(0.98,1.41)$ & 0.08 \\
Albumin $(\mathrm{g} / \mathrm{dL})<3.5$ & 2.03 & $(1.68,2.46)$ & $<0.001$ \\
Alkaline Phosphatase $(\mathrm{IU} / \mathrm{L})>=100$ & 1.28 & $(1.07,1.52)$ & 0.006 \\
\hline ECOG Eastern Cooperative Oncology & Group performance status, $(g / d L)$ \\
grams per decilitre, (IU/L) international units per litre & \\
\hline
\end{tabular}

serum alkaline phosphatase $>135 \mathrm{IU} / \mathrm{L}$ and carcinoembryonic antigen greater than $10 \mathrm{ng} / \mathrm{ml}$. Seamen et al. ${ }^{22}$ studied 90 patients with metastatic renal cell cancer and found that the presence of elevated alkaline phosphatase ( $>100 \mathrm{IU} / \mathrm{L})$ and/or bone pain was able to identify 27 of 28 patients with bone metastases identified on radionucleotide bone scan. In this study, the finding of elevated alkaline phosphatase in patients with multiple spine metastases, non-spine metastasis, and visceral metastasis supports both the pathophysiology of alkaline phosphatase and the findings of previous studies. The additional finding of no significant association with the presence of brain metastases served as an important control as the elevation of alkaline phosphatase was specific to bone and visceral metastases.

Previous studies have also examined serum alkaline phosphatase as a prognostic marker in malignancy. Gu et al. ${ }^{23}$ conducted a meta-analysis of eleven osteosarcoma studies between 1993 and 2013 with 1336 patients and found stable pooled hazard ratios confirming that elevated serum alkaline phosphatase was associated with poor survival. Manola et al. ${ }^{24}$ conducted a pooled 25-year analysis of 1362 metastatic melanoma patients from eight Eastern Cooperative Oncology Group trials and identified alkaline phosphatase as predictive of poor survival on proportional hazards modelling. Berry et al., Emrich et al. and Kantoff et al. identified serum alkaline phosphatase as a prognostic biomarker in metastatic prostate cancer and Halabi et al. developed a nomogram for survival probability in metastatic hormonerefractory prostate cancer including serum alkaline phosphatase. $^{25-28}$ Serum alkaline phosphatase was recently studied as risk factor for 30-day postoperative mortality in spinal metastatic disease patients in the National Surgical Quality Improvement Program (NSQIP), a database of short-term postoperative outcomes in North America. ${ }^{20}$ This database is fairly limited for oncologic studies because it lacks follow-up beyond 30-days and does not include important prognostic factors such as primary tumour histology, history of radiation, history of systemic therapy, visceral metastases, previous systemic therapy or history of local radiation. ${ }^{29}$ The findings of the present study extend the findings of prior studies of alkaline phosphatase in metastatic disease and establish a role for short and long-term prognostication in spinal metastatic disease. 
Previous prognostic studies of spinal metastatic disease patients have identified preoperative haemoglobin, ${ }^{30}$ white blood cell count, ${ }^{30}$ absolute neutrophil-to-lymphocyte ratio, ${ }^{31}$ platelet-tolymphocyte ratio, ${ }^{31}$ calcium, $^{32}$ and preoperative albumin ${ }^{32-34}$ as laboratory markers for postoperative mortality and morbidity. In this population of spinal metastatic disease patients, serum alkaline phosphatase was collected as part of routine liver function tests in greater than $70 \%$ of patients in the 30 -days before surgery. This existing collection of serum alkaline phosphatase but underutilisation for prognostication highlights an opportunity for the findings of this study to suggest new avenues for more efficiently using existing healthcare resources and improving the value of care delivery in metastatic disease.

Furthermore, well established scoring systems such as the Bauer, ${ }^{35}$ New England Spinal Metatasis, ${ }^{36}$ Katagiri, $^{37}$ Sioutos, ${ }^{7}$ SORG, ${ }^{38}$ Tokuhashi, $^{39}$ Tomita, $^{40}$ van der Linden ${ }^{41}$ and others ${ }^{6,42}$ currently incorporate measures of preoperative metastatic tumour burden by assessing the number of spine metastasis, other nonspine bone metastasis and visceral metastasis. However, despite controlling for these and other factors, serum alkaline phosphatase remained an independent prognostic factor on multivariable analysis; in addition, visceral metastasis no longer reached significance after incorporation of serum alkaline phosphatase. This suggests that existing scoring systems in spinal metastatic disease should be updated by additionally considering preoperative serum alkaline phosphatase as a candidate predictor.

There are several limitations to this study. The patient population was drawn from a single region and two tertiary care centres. Additionally, this study was retrospective and prospective validation of serum alkaline phosphatase as independent prognostic factor remains to be undertaken. All patients in this study underwent initial definitive surgical intervention for metastatic disease and the role of alkaline phosphatase in spinal metastatic disease managed with only radiotherapy, chemotherapy or palliative care remains to be determined. In addition, antiresorptive therapy in metastatic bone disease decreases adverse skeletal-related events, such as pathologic fractures and spinal cord compression, and is reflected by reductions in biomarkers of bone turnover including alkaline phosphatase. ${ }^{43,44}$ In patients receiving preoperative anti-resorptive therapy, elevated serum alkaline phosphatase may be a marker for missing response to anti-resorptive agents. As such, future studies of spinal metastatic disease patients undergoing operative intervention should further explore preoperative treatment with anti-resorptive therapy in relation to serum alkaline phosphatase levels at presentation as well as the role for postoperative prognostication on the basis of response to this administered therapy.

Nonetheless, this study identified serum alkaline phosphatase as an independent prognostic factor for overall survival in patients undergoing surgery for spinal metastatic disease and provided greater understanding of the relationship between this readily available laboratory marker and preoperative metastatic tumour burden. Future studies should consider assessment of this marker in the creation of nomograms and prediction models for spinal metastatic disease.

\section{CONCLUSION}

Serum alkaline phosphatase is associated with preoperative metastatic tumour burden (spine, bone, visceral) and is a biomarker for overall survival in spinal metastatic disease. Future studies building prognostic models for spinal metastatic disease should consider assessment of this simple preoperative biomarker.

\section{ACKNOWLEDGEMENTS}

The authors would like to acknowledge and thank the Orthopaedic Surgery Department and research team at Massachusetts General Hospital for administrative support.

\section{AUTHOR CONTRIBUTIONS}

A.V.K.: data collection, data analysis, manuscript writing. Q.T.: data collection, manuscript drafting. M.K.: data collection, manuscript writing. P.O.: data collection, manuscript writing. M.L.S.; study supervision, critical revision; J.H.S.: study supervision, study design, manuscript drafting and critical revisions.

\section{ADDITIONAL INFORMATION}

Supplementary information is available for this paper at https://doi.org/10.1038/ s41416-019-0407-8.

\section{Competing interests: The authors declare no competing interests.}

Ethics approval and consent to participate: This study was approved by the Partners Healthcare (Massachusetts General Hospital and Brigham and Women's Hospital) institutional review board and was performed in accord with the Declaration of Helsinki. Individual patient consent was waived as this study was restricted to retrospective review of de-identified patients only.

Data availability: All data generated or analysed during this study are included in this published article. The corresponding author of this study will accept reasonable requests for data that support the findings of this study.

Note: This work is published under the standard license to publish agreement. After 12 months the work will become freely available and the license terms will switch to a Creative Commons Attribution 4.0 International (CC BY 4.0).

Publisher's note: Springer Nature remains neutral with regard to jurisdictional claims in published maps and institutional affiliations.

\section{REFERENCES}

1. Sciubba, D. M. et al. Diagnosis and management of metastatic spine disease: a review. J. Neurosurg.: Spine 13, 94-108 (2010).

2. Wong, D. A., Fornasier, V. L. \& MacNab, I. Spinal metastases: the obvious, the occult, and the impostors. Spine 15, 1-4 (1990).

3. Harel, R. \& Angelov, L. Spine metastases: current treatments and future directions. Eur. J. Cancer 46, (2696-2707 (2010).

4. Heary, R. F. \& Bono, C. M. Metastatic spinal tumors. Neurosurg. Focus 11, 1-9 (2001).

5. Choi, D. et al. Prediction of quality of life and survival after surgery for symptomatic spinal metastases: a multicenter cohort study to determine suitability for surgical treatment. Neurosurgery 77, 698-708 (2015).

6. Leithner, A. et al. Predictive value of seven preoperative prognostic scoring systems for spinal metastases. Eur. Spine J. 17, 1488-1495 (2008).

7. Sioutos, P. J., Arbit, E., Meshulam, C. F. \& Galicich, J. H. Spinal metastases from solid tumors. Anal. Factors Affect. Surviv. Cancer 76, 1453-1459 (1995).

8. Coleman, J. E. Structure and mechanism of alkaline phosphatase. Annu. Rev. Biophys. Biomol. Struct. 21, 441-483 (1992).

9. Golub, E. E. \& Boesze-Battaglia, K. The role of alkaline phosphatase in mineralization. Curr. Opin. Orthop. 18, 444-448 (2007).

10. Mundy, G. R. Metastasis: Metastasis to bone: causes, consequences and therapeutic opportunities. Nat. Rev. Cancer 2, 584 (2002).

11. Gutman, A. B. Serum alkaline phosphatase activity in diseases of the skeletal and hepatobiliary systems: a consideration of the current status. Am. J. Med. 27 875-901 (1959)

12. Roodman, G. D. Mechanisms of bone metastasis. N. Engl. J. Med. 350, 1655-1664 (2004).

13. McShane, L. M. et al. REporting recommendations for tumor MARKer prognostic studies (REMARK). Breast Cancer Res. Treat. 100, 229-235 (2006).

14. Collins, G. S., Reitsma, J. B., Altman, D. G. \& Moons, K. G. Transparent reporting of a multivariable prediction model for individual prognosis or diagnosis (TRIPOD): the TRIPOD statement. BMC Med. 13, 1 (2015).

15. Quan, $\mathrm{H}$. et al. Updating and validating the Charlson comorbidity index and score for risk adjustment in hospital discharge abstracts using data from 6 countries. Am. J. Epidemiol. 173, 676-682 (2011).

16. Katagiri, $H$. et al. New prognostic factors and scoring system for patients with skeletal metastasis. Cancer Med. 3, 1359-1367 (2014).

17. Stekhoven, D. J. \& Bühlmann, P. MissForest-non-parametric missing value imputation for mixed-type data. Bioinformatics 28, 112-118 (2011).

18. López-Ratón, M., Rodríguez-Álvarez, M. X., Cadarso-Suárez, C. \& Gude-Sampedro, F. OptimalCutpoints: an R package for selecting optimal cutpoints in diagnostic tests. J. Stat. Softw. 61, 1-36 (2014). 
19. Posada, D. \& Buckley, T. R. Model selection and model averaging in phylogenetics: advantages of Akaike information criterion and Bayesian approaches over likelihood ratio tests. Syst. Biol. 53, 793-808 (2004).

20. Karhade, A. V., Thio, Q. C., Ogink, P. T. \& Schwab, J. H. Serum alkaline phosphatase and 30-day mortality after surgery for spinal metastatic disease. J. Neurooncol. 140, 165-171 (2018).

21. Tartter, P. I., Slater, G., Gelernt, I. \& Aufses, A. H. Jr Screening for liver metastases from colorectal cancer with carcinoembryonic antigen and alkaline phosphatase. Ann. Surg. 19, 357 (1981).

22. Seaman, E., Goluboff, E. T., Ross, S. \& Sawczuk, I. S. Association of radionuclide bone scan and serum alkaline phosphatase in patients with metastatic renal cell carcinoma. Urology 48, 692-695 (1996).

23. Gu, R. \& Sun, Y. Does serum alkaline phosphatase level really indicate the prognosis in patients with osteosarcoma? A meta-analysis. J. Cancer Res. Ther. 14 (Supplement), S468-S472 (2018).

24. Manola, J., Atkins, M., Ibrahim, J. \& Kirkwood, J. Prognostic factors in metastatic melanoma: a pooled analysis of Eastern Cooperative Oncology Group trials. J. Clin. Oncol. 18, 3782-3793 (2000).

25. Halabi, S. et al. Prognostic model for predicting survival in men with hormonerefractory metastatic prostate cancer. J. Clin. Oncol. 21, 1232-1237 (2003).

26. Emrich, L. J., Priore, R. L., Murphy, G. P. \& Brady, M. F. Prognostic factors in patients with advanced stage prostate cancer. Cancer Res. 45, 5173-5179 (1985).

27. Berry, W. R., Laszlo, J., Cox, E., Walker, A. \& Paulson, D. Prognostic factors in metastatic and hormonally unresponsive carcinoma of the prostate. Cancer $\mathbf{4 4}$, 763-775 (1979).

28. Kantoff, P. W. et al. Hydrocortisone with or without mitoxantrone in men with hormone-refractory prostate cancer: results of the cancer and leukemia group B 9182 study. J. Clin. Oncol. 17, 2506-2513 (1999).

29. Karhade, A. V., Larsen, A. M., Cote, D. J., Dubois, H. M. \& Smith, T. R. National databases for neurosurgical outcomes research: options, strengths, and limitations. Neurosurgery 83, 333-344 (2018).

30. Pereira, N. R. P. et al. Development of a prognostic survival algorithm for patients with metastatic spine disease. JBJS 98, 1767-1776 (2016).

31. Thio, Q., et al. Prognostic role of neutrophil-to-lymphocyte ratio and platelet-tolymphocyte ratio in patients with bone metastases. Br. J. Cancer 119, 737-743 (2018).
32. Ogihara, S. et al. Prognostic factors for patients with spinal metastases from lung cancer. Spine 31, 1585-1590 (2006).

33. Ghori, A. K. et al. Modeling 1-year survival after surgery on the metastatic spine. Spine J. 15, 2345-2350 (2015).

34. Schoenfeld, A. J. et al. Assessing the utility of a clinical prediction score regarding 30-day morbidity and mortality following metastatic spinal surgery: the New England Spinal Metastasis Score (NESMS). Spine J. 16, 482-490 (2016).

35. Bauer, H. C. \& Wedin, R. Survival after surgery for spinal and extremity metastases: prognostication in 241 patients. Acta Orthop. Scand. 66, 143-146 (1995).

36. Schoenfeld, A. J. et al. Assessing the utility of a clinical prediction score regarding 30-day morbidity and mortality following metastatic spinal surgery: the New England Spinal Metastasis Score (NESMS). Spine J. 16, 482-490 (2016).

37. Katagiri, H. et al. Prognostic factors and a scoring system for patients with skeletal metastasis. J. Bone Jt. Surg. Br. 87, 698-703 (2005).

38. Paulino Pereira, N. R. et al. The SORG nomogram accurately predicts 3- and 12months survival for operable spine metastatic disease: external validation. J. Surg. Oncol. 115, 1019-1027 (2017).

39. Tokuhashi, Y., Matsuzaki, H., Toriyama, S., Kawano, H. \& Ohsaka, S. Scoring system for the preoperative evaluation of metastatic spine tumor prognosis. Spine 15, 1110-1113 (1990)

40. Tomita, K. et al. Surgical strategy for spinal metastases. Spine 26, 298-306 (2001).

41. van der Linden, Y. M., Dijkstra, S. P., Vonk, E. J., Marijnen, C. A. \& Leer, J. W. H., Group DBMS. Prediction of survival in patients with metastases in the spinal column: results based on a randomized trial of radiotherapy. Cancer 103, 320-328 (2005).

42. Ahmed, A. K., et al. Predicting survival for metastatic spine disease: a comparison of nine scoring systems. Spine J. 18, 1804-1814 (2018).

43. Lipton, A. et al. Pamidronate prevents skeletal complications and is effective palliative treatment in women with breast carcinoma and osteolytic bone metastases: long term follow-up of two randomized, placebo-controlled trials. Cancer 88, 1082-1090 (2000).

44. Henry, D. H. et al. Randomized, double-blind study of denosumab versus zoledronic acid in the treatment of bone metastases in patients with advanced cancer (excluding breast and prostate cancer) or multiple myeloma. J. Clin. Oncol. 29, 1125-1132 (2011). 